\title{
Understanding Context Before Using It
}

\author{
Mary Bazire $^{1}$ and Patrick Brézillon ${ }^{2}$ \\ ${ }^{1}$ Laboratoire Cognition \& Usages, CNRS FRE 2627, \\ 2 rue de la Liberté 93523 Saint-Denis Cedex \\ mary.bazire@cognition-usages . org \\ ${ }^{2}$ LIP6, Case 169, Université Paris 6, \\ 8 rue du Capitaine Scott, 75015 Paris, France \\ Patrick.Brézillon@lip6.fr
}

\begin{abstract}
This paper presents an attempt to point out some problematic issues about the understanding of context. Although frequently used in cognitive sciences or other disciplines, context stays a very ill-defined concept. Our goal is to identify the main components of the context on the basis of the analysis of a corpus of 150 definitions coming mainly from the web in different domains of cognitive sciences and close disciplines. We analyzed this corpus of definitions through two methods, namely LSA [1], [2] and STONE [3], [4], and we conclude that finally the content of all the definitions can be analyzed in terms of few parameters like constraint, influence, behavior, nature, structure and system.
\end{abstract}

Keywords: Context, Definition, Contextual elements.

\section{Introduction}

Etymologically, context of a given utterance (= co-text) is composed by the part of text before [5] and after the utterance (cf. textual linguistics). The meaning of the term knew an evolution towards a larger acceptation and now the meaning generally accepted is that context is the set of circumstances that frames an event or an object. This concept is increasingly used in a large number of disciplines like psychology, especially since the emergence of situated cognition theories [6], those theories considering cognition in its natural context [7].

However, it is difficult to find a relevant definition satisfying in any discipline. Is context a frame for a given object? Is it the set of elements that have any influence on the object? Is it possible to define context a priori or just state the effects a posteriori? Is it something static or dynamic? Some approaches emerge now in Artificial Intelligence (e.g. see [8]). In Psychology, we generally study a person doing a task in a given situation. Which context is relevant for our study? The context of the person? The context of the task? The context of the interaction? The context of the situation? When does a context begin and where does it stop? What are the real relationships between context and cognition? 
Hereafter, the paper is organized in the following manner. Section 2 shows the key role plays by context in psychology. Section 3 presents the importance to elaborate a robust of context for an effective use of this concept. Section 4 introduces briefly the two techniques of semantic analysis (the Latent Semantic Analysis [1], [2] and STONE [3], [4]) that have been applied to a corpus of definitions of context for identifying more clearly the concept of context. Finally, Section 5, proposes a first model taking into account every component of the context and presents an efficient definition for addressing psychological problems.

\section{Context, a Fundamental Notion in Psychology}

There are a number of concepts in Psychology that are considered to be close to context, such as the notions of situation and field (e.g. in the Witkin theory on cognitive styles [9]), the notion of milieu (e.g. in animal psychology), the notion of distractor (e.g. in perception psychology), background (e.g. in the gestalt theory). All these notions are considered as parts of the immediate context of a stimulus. References on context exist in all the different branches of psychology. This is certainly the most used concept [10]. In general psychology, context is often used in the sense of "set of situational elements in which the object (i.e. stimulus considered above) being processed is included" [5]. Context has multiple effects of interest in Psychology: studies on memorization and lexical access processes point out some priming phenomenon (the primer belongs to the context and is a stimulus that affects previously the processing of the current one; for example, categorization [11] and attentional capacities [12] are affected by context.

Bastien [13] distinguishes two opposite views about the role of context in human cognition. The first view considers cognition as a set of general processes that modulate the instantiation of general pieces of knowledge by facilitation or inhibition. In the second view (in the area of situated cognition [6], [14], context has a more central role as a component of cognition by determining the conditions of knowledge activation as well as the limit of knowledge validity. These two opposite views underline that context may have an internal nature or an external one. On the one hand, context is an external object relative to a given object, when, on the other hand, context belongs to an individual and is an integral part of the representation that this individual is building of the situation where he is involved. According to this second viewpoint, "context cannot be separated from the knowledge it organizes, the triggering role context plays and the field of validity it defines" [13].

Those two opposite views could be reconciled in a prototype model [15]. Those seem to be two points of attraction "around which the various notions of context seem to converge: (1) a local point which is related to the structural environment. It is activated and constructed in the ongoing interaction as it becomes relevant [16], and is eventually shared by interactants; and (2) a global point, which refers to the given external components of the context. It includes knowledge and beliefs, and the general experience resulting from the interplay of culture and social community" [17]. 
Thus, context has an explanatory power in Psychology. The question then is to identify the main dimensions of context. We address this question in the next section.

\section{Need of a Consensual Definition}

Previous sections show that context is almost a buzzword in psychology, as in a number of other ones, such as computer science or linguistic. However, like some words as "concept" or "system", the word "context" either is not defined or it is possible to find as many definitions (in an ad hoc manner) as authors. A reason is that this word is used supposing that every body knows its meaning, otherwise the author wants to delineate the particular meaning he gives at this word. This epistemological problem [18], [19] will be not discussed in this paper. Moreover, one notes an increasingly use of this word on the Web: 5\% in 1997 and 15\% in 2004 of Web pages used this word, clearly not always in a useful sense (e.g. "In the context of this situation, we think that...") but more frequently now. When the word "context" is used in a meaningful sense, there is no real consensual definition, mainly because definitions of context are too much dependent of their own contexts (e.g. the discipline in which the definition is taken but also on both the kind and the goal of a given text). However, we think that few parameters may leads to an objective view (decontextualized view) of the definitions of context.

\subsection{The Corpus of Definition}

Brézillon [8], [18], [19] and Brun [20] collected a set of definitions of context, mainly on the Web. Initially the goal was to develop an effective model of context to be used in a knowledge-based system. After a while it appeared that it was not possible to develop in isolation a model of context because context, knowledge and reasoning [21] are strongly intertwined. Thus, the idea was to design and develop rather a software based on a context-based formalism of representation of knowledge and reasoning, formalism called Contextual Graphs [21]. For Brézillon [18], the lack of an operational definition explains several failures noted in knowledge based system use because (1) users and their contexts are not taken into account, (2) out of its context of validity, there is an incorrect use of the knowledge, (3) with the infinite number of contexts, it is not possible to endow prior its use a system with all the needed knowledge, and (4) computer systems have not the means to identify the context in which a user's request must be interpreted.

We now have a database of more than 150 definitions of context pick up on the Web from various disciplines such as computer science, philosophy, economy, business, HCI, etc. Each definition is entered in the database according to a frame containing:

- the definition

- the object about which context is defined

- the domain from which the definition has been found

- the reference (URL or bibliographical reference)

- eventual comments or complements to the definition. 
For example:

\section{Definition 2:}

Context is what constrains a step of a problem solving without Item: intervening in its explicitly

A step of a problem solving Domain:

Artificial Intelligence, Decision Support System Source:

Brézillon P. (1999) Context in problem solving: A survey. The Knowledge Engineering Review, 14(1): 1-34.

Comments:

The goal of this collection of definitions relies on the extraction of consensual, necessary or dependent features related to a given domain of knowledge. For instance, the definition "context is what constrains a problem solving without intervening in it explicitly" above is similar to the definition "All that may influence a given process whom first causes are known" [22]. By abstracting key elements of the definitions we thought to develop a resulting definition able to cover a large class of definitions. Among the different methods available, we chose to extract main components of the definitions with the software called STONE [3], [4]. In our approach we have taken only half of the corpus of definitions (indeed, 66 definitions) in order to use the second half for validation of our model (Selection of definitions has been made by classical statistical means).

However, most of the definitions in our initial corpus mostly belongs to cognitive sciences. The most represented areas in the database are: artificial intelligence (39), documentation (27), cognitive ergonomics (9), cognitive psychology (6), business (4), philosophy (2), linguistic (4) and one in education sciences, another in medicine and the last one in neuroscience (some definitions are multidisciplinary).

The definitions are collected in order to avoid redundancy in order to have, as much as possible, each definition original and unique. We have been obliged to put apart definitions coming from some highly specialized domains, such as logic or programming, because such definitions resulted in too much abstract, formal or specific definitions. The corpus presents nevertheless a sufficient variety: "Context is what constrained a problem solving without intervening in it explicitly" [18]; "Something that growths and change as a function of the time, user's requests and the growth intelligence of the system" [23]; "An ordered set of modules which can change during the process of proving a formula" [24], etc. This variety arises from the fact that there is no absolute context, context being relative to something. As a consequence, context must be studied according to its use.

\subsection{The Latent Semantic Analysis (LSA)}

Latent Semantic Analysis (LSA, in free access at http://lsa.colorado.edu) is a theory (a memory model) and a method (a mathematical model) used for extracting and representing the meaning of words in their context of use by applying statistical 
computations to very large corpus of texts [1], [2]. The representation of the meaning of a word or a sentence in LSA is given by a vector in a multi-dimensional space, as an average of the meanings of all the paragraphs where the word occurs (this point is detailed in [25]). The distance between two vectors coding two words or paragraphs represents a semantic distance between two terms. By choosing this method, we hypothesize that clusters of definitions semantically close could emerge.

The application of LSA to our corpus gives a correlation matrix that represents the semantic proximity between each paragraph (a definition in our corpus). The lower correlation was 0.27 for two definitions corresponding to "a set of constraints" and "a shared ontology which constitutes a shared vocabulary." The higher correlation was 0.93 for definitions including "what constrains each step of a problem solving without intervening in its explicitly." and "The set of the properties that are associated to an entity according to the environment in which is the entity." The two later definitions were the closest according to the LSA model. The correlation matrix being too large to treat manually, we apply then a cluster analysis.

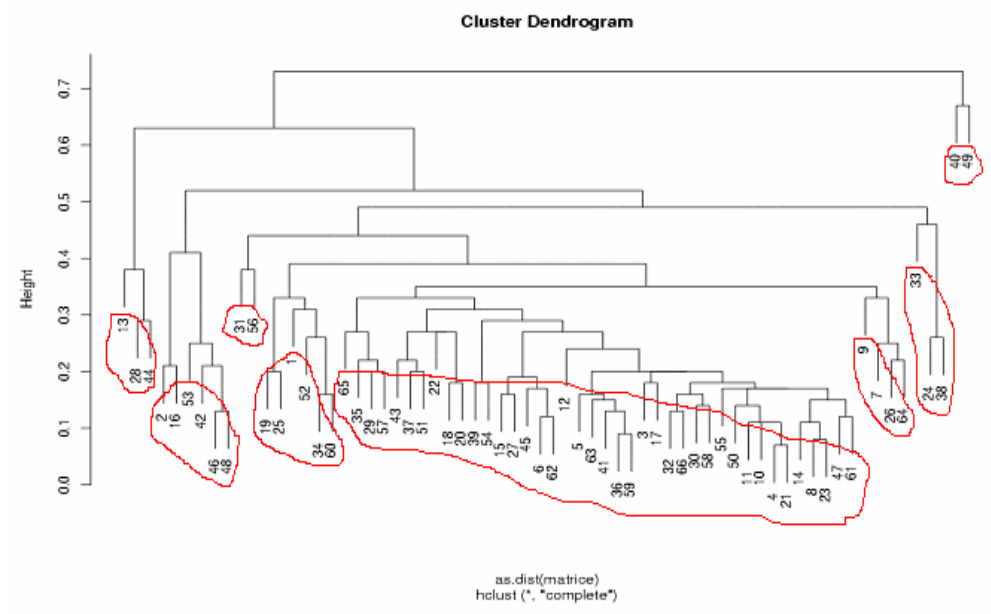

Fig. 1. Cluster analysis of 66 definitions of context applied to the correlation matrix

However, the cluster analysis does not provide relevant results for pointing out some particular clusters. The method allows to identify successive fitting and the marginality of some definitions as shown in Figure 1. First, it is possible to isolate two definitions, namely:

Definition 40: "Context is any identifiable configuration of environmental, mission-related, and agent-related features that has predictive power for behaviour." and

Definition 49: "A shared goal between instructor and students."

Marginality could appear from a too large specificity. Indeed, the two definitions are in-depth and are domain-dependent with respect to the knowledge. There is a problem of granularity and this opens the question of the management of different levels of 
generality in the corpus. Some definitions are at a high level of generality and could be applied directly across disciplines, when other definitions are specific of a given situation in a given domain, generally given as ad hoc definitions.

Therefore, it is possible to explain some groups of definitions in Figure 1. A first group concerns definitions around the idea of a psychological process of representation building. For Bastien [5], one of the main role of context is to remove ambiguity or to interpret situation. A second group meets together definitions about the spatial dimension of context. This is generally in this acceptance of the term that the popular speech uses the term of context: the context of an object is the set of physical elements in which the object is embedded. A third group gathers some definitions taken from linguistic. The other groups are difficult to identify.

The lack of clear result by the LSA approach leads us to try a second approach called STONE [3], [4]. It is a tool for the analysis by a binary questionnaire, i.e. the answering modalities must fit presence or lack of selected features. This method allows the representation of inter-modality associations by a set of implication relations, such as either a tree (with respect to the answering modality) or a Galois lattice (with respect to the set of answers given by one person. In such a representation, nodes represent object categories and edges represent relations of semantic inclusion [4], [3], [26].

A first step of our work was to extract manually concepts identified explicitly in the definitions with their logical implications. Then, according to the occurrence of the concepts found, STONE provides a tree giving the fit of the concepts as presented in Figure 2 for the component "Behavior" in the definitions of context, with the number of definitions concerned by this component.

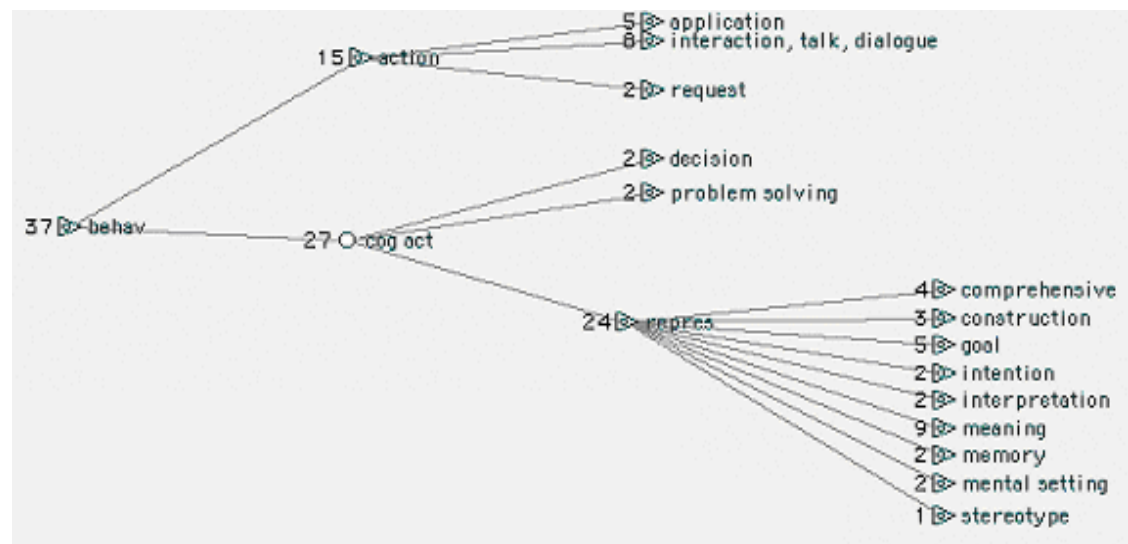

Fig. 2. An example of the context component "behavior"

The analysis of our corpus by STONE provides the following results. Six main components are identified--constraint, influence, behavior, nature, structure and system-and are then refined (for 4 of them) into more specific parts. The object concerned by a context can be an action or a cognitive activity. Its nature is external, 
internal (see details in [13]) or conditional. Context can be either a process (and thus dynamic) or an information (a concept, a data, a document, an entity, a piece of knowledge, a proposition, a set of propriety or a stimulus). Finally, context can have a structure of set or frame such as a network or an ontology, whose constituting elements share some relations or not.

More than half of the 66 definitions (37 definitions) concern the context of a behavior, the behavior being an action (applied to an application software, a language act or a request), or a cognitive activity (decision, problem solving, or representation construction). Such contexts are not external and objective physical contexts of an object because they intervene for the understanding, the goal determination, the intention, the construction of an interpretation, a meaning or a stereotype and for the memorization.

From these trees, STONE builds the Galois lattice for representing the implication relations shared by the definitions. For example, see Figure 3 the lattice corresponding to the context component "behavior".

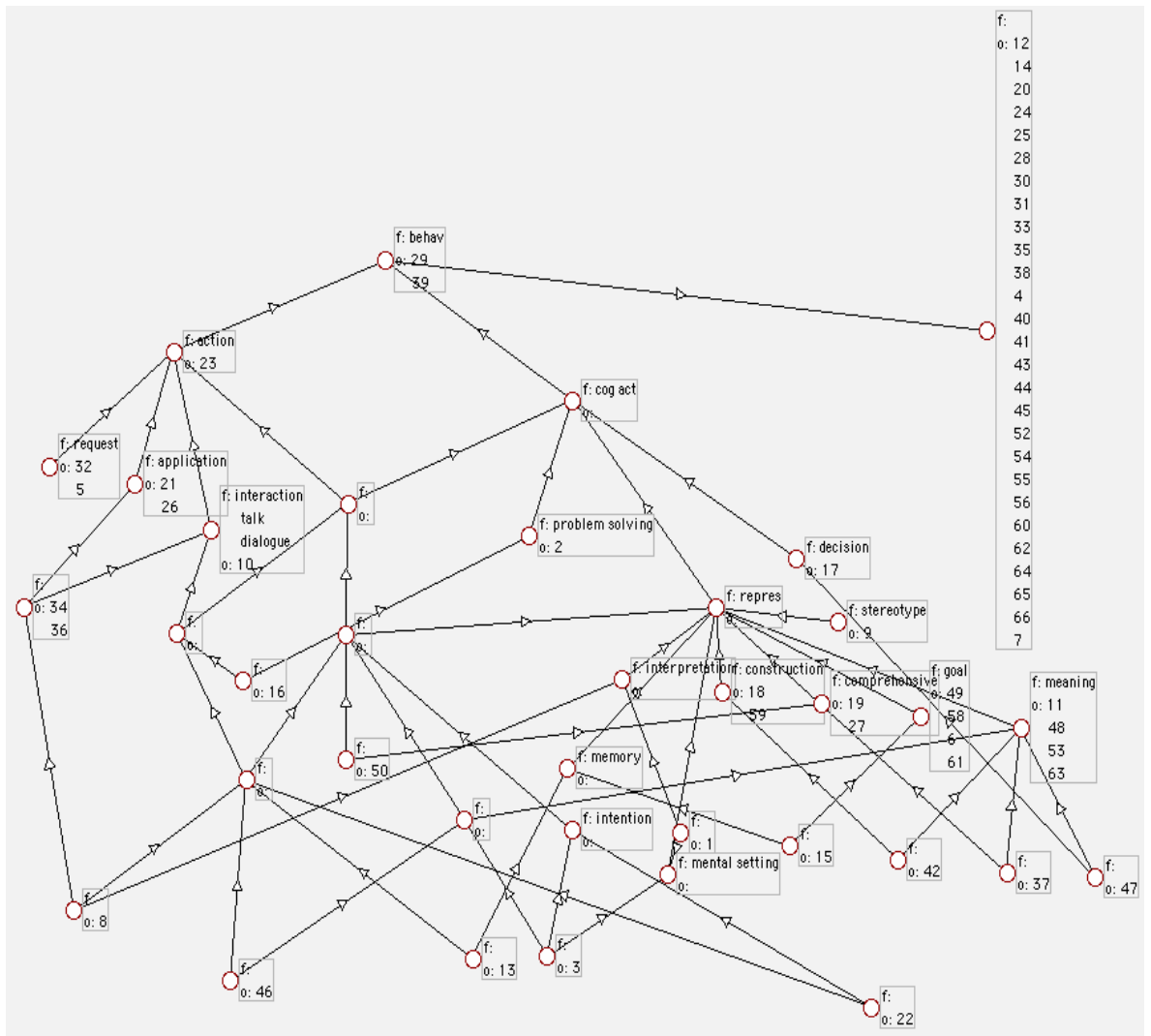

Fig. 3. Example of lattice: the context component "behavior" 
In such an analysis, the identified groups are characterized by the fact that some definitions share concepts in a more or less direct way. Thus, seven definitions of our corpus of 66 definitions mention the notion of meaning as a sort of representation, a sort of cognitive activity, or a sort of behavior. Nodes without neither label nor definition represent categories without any example. Such nodes are generally intermediate and with more than one super-ordinate node. Moreover, the lattice defines relations as implication relations between definitions: nodes representing categories (action, cognitive activity, decision, for instance) and edges representing relations of semantic inclusion. Consequently, the following two definitions:

Definition 34: "Context is any information that characterizes a situation related to the interaction between humans, applications and the surrounding environment", and

Definition 36: "Context is any information that can be used to characterize the situation of an entity, where the entity is a person, place, or object that is considered relevant to the interaction between a user and its application, including the user and the application themselves.."

belong to the same category and their subordinate categories are the categories "application" and "interaction, dialogue, talk", themselves, subordinates to "action." which is a kind of behavior. Some categories make obviously a choice between the two main sub-categories (the observed behavior is either an action or a cognitive activity), whereas other definitions, such as the definition:

Definition 46: "Context is the interrelated conditions in which something exists or occurs, and the parts of a discourse or treatise that precede and follow a special passage and may fix its true meaning"

takes the two alternatives into account. Nevertheless, it happens that the lattice become very complex if a definition mentions a great deal of proprieties. For example, the definition:

Definition 8: "Context is any information that can be used to characterize and interpret the situation in which a user interacts with an application at a certain time."

may be too explicit because it mentions the ideas of application software, interaction, cognitive activity, problem solving, interpretation and representation. Such an accuracy in the definition make the lattice complex. Conversely, excluding such definitions could show only the significant relations, notably relations that concern more than one definition. There is a compromise to find here.

The two analysis used (LSA and STONE) reveal the diversity (almost a heterogeneity) showed by the collected definitions. Indeed, the mixing up of the lattices and the progressive exclusion of definitions in the cluster analysis illustrates this situation.

Note that the grouping in clusters of linguistic definitions confirms that a definition of context can be very dependent on the discipline it belongs to (see the grouping in clusters of the linguistic definitions). 


\section{A Working Model for Context}

Our conclusions of the corpus study lead us to build a model of context representing the components of a situation (where the context is taken into account) and the different relations between those components, with the hypothesis that the reason why definitions diverge is that they don't put their focus of attention on the same topics.

Then, the topic on which definitions put the focus on may allow discriminating definitions. A situation could be defined by a user, an item in a particular environment, and eventually an observer (according to certain definitions). Context interferes with each of these elements as illustrated in Figure 3. Some definitions focus on certain relations, when others concern other relations.

We separate context and environment because we consider that the physical environment is not totally relevant in a task running and we propose that context represents all that it is significant at a given moment but can belong to any of the terms of our model, depending to the task goal.

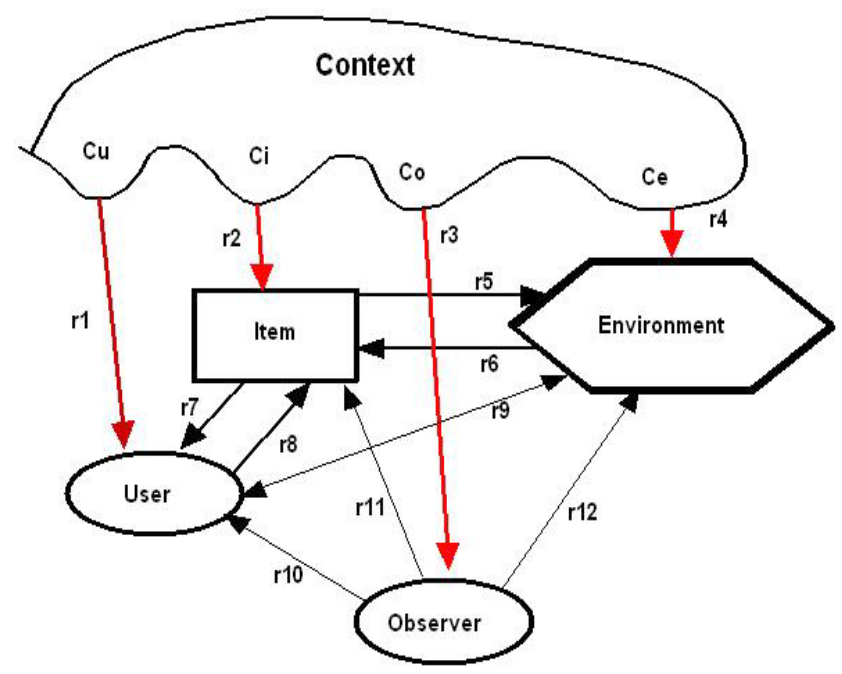

Fig. 4. Our proposal for a model of context

For example, consider the two following definitions.

Definition 2: "Context is what constrains each step of a problem solving without intervening in its explicitly."

The focus is here on the item (a step of a problem solving) and the goal is to solve the problem at this step. In Figure 4, the definition takes into account the context of the item $(\mathrm{Ci})$, the user and the environment (the other steps of the problem solving), eventually the relations R6 and R8. The other topics are not considered. The definition: 
Definition 57: "Context has two dimensions: (1) Ecology: aspects of the school that are not living, but nevertheless affect its inhabitants (resources available, policies and rules, and size of the school); and (2) Culture to capture the informal side of schools."

focuses on life in a school (the item in Figure 4). The goal is here to integrate a school in the neighborhood of a city. Here, the important topics are the user, the contexts of the item and of the environment and the relations R5, R6, R2, R4, R7, R8 and R9.

We hope that this model will allow us to build groups regarding to the topics they focus on. Then, in order to work on a definition of context, we would concentrate our work first on the definitions that focus on context itself. We hypothesize also that the differences between domains could be explain in those terms: by the topics they single out.

Another study, lead concurrently concerned the necessary components of a situation in order to be easily understood and agreed with [27], allows us to consider that context can be specified for a given situation by the answering of the following questions : Who? What? Where? When? Why? and How? "Who" indicates the subject, the agent of the action. "What" represents the object, the patient that sustains the action. "Where" and "When" give information about the spatio-temporal location of the considered action. "Why" gives the intentions, the goal (and eventually the emotions) of the subject. At last, "How" makes explicit the procedure needed to realize the action. Our study mentioned above showed that the optimal order of presentation is Action-Object-Agent-Location, which gives a primordial role to action that confirms the necessity to consider the goal as a part of context.

\section{Conclusion}

Our analysis of definitions of context collected on the Web shows that context could be analyzed through six essential components. The context acts like a set of constraints that influence the behavior of a system (a user or a computer) embedded in a given task. We discuss the nature and structure of context in the definitions. There is no consensus about the following questions: Is context external or internal? Is context a set of information or processes? Is context static or dynamic? Is context a simple set of phenomenon or an organized network? Indeed, the two analysis used in this study (LSA and STONE) point out the fundamental role played by the context in the process of representation construction. Finally we can say that context occurs like what is lacking in a given object for a user to construct a correct representation. We state that a definition of context depends on the field of knowledge that it belongs to.

Concurrently to these two analysis, an other one, more qualitative (by hand), leads us to extract some determining factors in a definition of context: the entity concerned by the context, its focus of attention, its activity, its situation, its environment and eventually, an observer.

Now, we plan to validate our results on the whole corpus of 150 definitions. However, this study offers a contribution on the cohesiveness of the different approaches met in the literature (e.g. for modeling reasoning, ubiquitous computing and in context-aware applications). In this sense, we think to have bring a new insight. A possible extension of this work would be to take into account the dynamic 
dimension of context through the definitions of context because we are convinced that if context is often considered in a passive way, context has a real active nature (its dynamical properties are not systematically shown). We hope that this work can be useful as a beginning for the community to discuss the meaning of the term.

\section{References}

1. Landauer, T. \& Dumais, S. (1997) A solution to Plato's problem: The Latent Semantic Analysis Theory of Acquisition, Induction and Representation of Knowledge. Psychological Review. 104: 211-240.

2. Landauer, T., Foltz, P. \& Laham, D. (1998) An introduction to Latent Semantic Analysis. Discourse Processes. 25 : 259-284.

3. Poitrenaud, S. (2001) Complexité cognitive des interactions Homme-Machine. Paris: L'Harmattan.

4. Bernard, J.-M. \& Poitrenaud, S. (1999) L'analyse implicative bayésienne multivariée d'un questionnaire binaire: quasi-implications et treillis de Galois simplifié. Math. Inf. Sci. hum. 147: 25-46.

5. Bastien, C. (1998) Contexte et situation In Houdé, O., Kayser, D., Koenig, O., Proust, J. \& Rastier, F., Dictionnaire des Sciences Cognitives. Paris: PUF.

6. Clancey, W. (1994) Situated Cognition: How representations are created and given meaning, In Lewis, R. and Mendelsohn, P., Eds. Lessons from learning. Amsterdam. pp 231-242.

7. Seifert, C. (1999) Situated cognition and learning. In Wilson, R. \& Keil, F. The MIT encyclopedia of the cognitive sciences. Cambridge: The MIT Press.

8. Brézillon, P. (2003) Representation of procedures and practices in Contextual graphs. The Knowledge Engineering Review, 18(2): 147-174.

9. Witkin, H.A., Goodenough, D.R. \& Oltman, P.K. (1979) Psychological differenciation current status, J. Person. Soc. Psychol., 37 : 1127-1146.

10. Péreira, F. \& Quelhas, A. C. (1998) Cognition and context: introductory notes. In Quelhas, A. C. \& Péreira, F., Cognition and Context. Analise psicologica: Lisbonnes.

11. Barsalou, L. (1982) Context-independent and context-dependent information in concepts. Memory \& Cognition. 10(1): 82-93.

12. Sabri, M., Melara, R. \& Algom, D. (2001) A confluence of contexts: Asymmetric versus global failures of selective attention to Stroop dimensions. Journal of experimental psychology: Human perception and performance. 27(3): 515-537.

13. Bastien, C. (1999) Does context modulate or underlie human knowledge? In Quelhas, A.C. \& Péreira, F., Cognition and Context. Analise psicologica: Lisbonnes.

14. Newell, A. \& Simon, H. (1972) Human Problem Solving. Prentice-Hall, Inc. : Englewood Cliffs.

15. Rosch, E. H. (1978) Principles of categorization. In: E. Rosch \& B. Lloyd, eds., Cognition and Categorization. Hillsdale, N.J.: Erlbaum Associates. 27-48.

16. Sperber, D. \& Wilson, D. (1986). Relevance: Communication and Cognition. Cambridge, MA: MIT Press.

17. Akman V., Bazzanella C. (2003) The complexity of context, in Varol Akman, Carla Bazzanella(eds.) On Context, Journal of Pragmatics, special issue, 35, 321-329

18. Brézillon, P. (2002) Modeling and using context: Past, present and future. Rapport de recherche interne LIP6, Paris. 
19. Brézillon, P. (1999) Context in Problem solving: A Survey. The Knowledge Engineering Review. 14(1): 1-34.

20. Brun, V. (2002) Mémoire de maîtrise: Université de Paris 8.

21. Brézillon, P., (2005) Task-realization models in Contextual Graphs. Modeling and Using Context (CONTEXT-05), A. Dey, B.Kokinov, D.Leake, R.Turner (Eds.), Springer Verlag, LNCS (This volume).

22. Denis, M. \& Sabah, G. (1993) In Modèles et concepts pour la science cognitive. Hommage à J.-J. Le Ny. (Grenoble: PUG).

23. Minsky, M. (1986) The Society of Mind, Picador Publisher.

24. Bielikova, M. \& Navrat, P. (1997) A multilevel knowledge representation of strategies for combining modules. Proceedings of the Seventh International Conference on AI and Information-Control Systems of Robots. World Scientific, Singapore: 155-168.

25. Bazire, M. (2003) Effets des composantes du contexte sur la compréhension. Mémoire de DEA: Université de Paris 8.

26. Tijus, C. (2001) Contextual categorization and cognitive phenomena, P.B.V. Akman, R. Thomason, R.A. Young (Eds) Modeling and Using Context. Third International and Interdisciplinary Conference, CONTEXT, 2001.

27. Jang, S. \& Woo, W. (2003) Ubi-UCAM : A unified context-aware application model. Proceedings of the 4th international and interdisciplinary conference CONTEXT 2003. Stanford : P. Blackburn et al. (Eds). pp.178-189. 